558.

\title{
A GEOMETRICAL INTERPRETATION OF THE EQUATIONS OB- TAINED BY EQUATING TO ZERO THE RESULTANT AND THE DISCRIMINANTS OF TWO BINARY QUANTICS.
}

[From the Proceedings of the London Mathematical Society, vol. v. (1873-1874), pp. 31-33. Read March 12, 1874.]

Consider the equations

$$
\begin{aligned}
& U=(a, b, \ldots \gamma t, 1)^{\lambda}=0, \\
& U^{\prime}=\left(a^{\prime}, b^{\prime}, \ldots \gamma t, 1\right)^{\lambda^{\prime}}=0 ;
\end{aligned}
$$

and equating to zero the discriminants of the two functions respectively, and also the resultant of the two functions, let the equations thus obtained be

$$
\begin{aligned}
& \Delta=(a, b, \ldots)^{2 \lambda-2}=0, \\
& \Delta^{\prime}=\left(a^{\prime}, b^{\prime}, \ldots\right)^{2 \lambda^{\prime}-2}=0, \\
& R=(a, b, \ldots)^{\lambda^{\prime}}(\dot{a}, b, \ldots)^{\lambda}=0 .
\end{aligned}
$$

I take $(a, b, \ldots),\left(a^{\prime}, b^{\prime}, \ldots\right)$ to be linear functions of the coordinates $(x, y, z)$; and $t$ to be an indeterminate parameter. Hence $U=0$ represents a line the envelope whereof is the curve $\Delta=0$, or, what is the same thing, the equation $U=0$ represents any tangent of the curve $\Delta=0$; this is a unicursal curve of the order $2 \lambda-2$ and class $\lambda$, with $3(\lambda-2)$ cusps and $\frac{1}{2}(\lambda-2)(\lambda-3)$ nodes. Similarly $U^{\prime}=0$ represents a line the envelope of which is the curve $\Delta^{\prime}=0$ : this is a unicursal curve of the order $2 \lambda^{\prime}-2$ and class $\lambda^{\prime}$, with $3\left(\lambda^{\prime}-2\right)$ cusps and $\frac{1}{2}\left(\lambda^{\prime}-2\right)\left(\lambda^{\prime}-3\right)$ nodes; the equation $U^{\prime}=0$ represents any tangent of this curve.

The equations $U=0, U^{\prime}=0$ considered as existing simultaneously with the same value of $t$, establish a $(1,1)$ correspondence between the tangents (or if we please, between the points) of the two curves. The locus of the intersection of the corre- 
sponding tangents is the curve $R=0$, a unicursal curve of the order $\lambda+\lambda^{\prime}$, with $\frac{1}{2}\left(\lambda+\lambda^{\prime}-1\right)\left(\lambda+\lambda^{\prime}-2\right)$ nodes and no cusps; consequently of the class $2\left(\lambda+\lambda^{\prime}-1\right)$.

It is to be shown that the curve $R=0$ touches the curve $\Delta=0$ in $\lambda^{\prime}+2 \lambda-2$ points, and similarly the curve $\Delta^{\prime}=0$ in $2 \lambda^{\prime}+\lambda-2$ points.

In fact, consider any tangent $T^{\prime}$ of the curve $\Delta^{\prime}$; let this meet the curve $\Delta$ in a point $A$, and let $Q$ be the tangent at $A$ to the curve $\Delta$; suppose, moreover, that $T$ is the tangent of $\Delta$ corresponding to the tangent $T^{\prime}$ of $\Delta^{\prime}$. Then if $Q$ and $T$ coincide, the corresponding tangent of $T^{\prime}$ will be $Q$, and the curve $R$ will pass through $A$. It is easy to see that in this case the curves $R, \Delta$ will touch at $A$. Again, if $P$ be a tangent from $A$ to the curve $\Delta$, then, if $P$ and $T$ coincide, the corresponding tangent of $T^{\prime}$ will be $P$, and the curve $R$ will pass through $A$; but in this case the point $A$ will be a mere intersection, not a point of contact, of the two curves.

The tangents $T, Q$ each correspond to $T^{\prime}$, and they consequently correspond to each other. For a given position of $T$ we have a single position of $T^{\prime}$, and therefore $2 \lambda-2$ positions of $A$, or, what is the same thing, of $Q$; that is, for a given position of $T$ we have $2 \lambda-2$ positions of $Q$. Again, to a given position of $Q$ corresponds a single position of $A$, therefore $\lambda^{\prime}$ positions of $T^{\prime}$, therefore also $\lambda^{\prime}$ positions of $T$; that is, for a given position of $Q$ we have $\lambda^{\prime}$ positions of $T$. The correspondence between $T, Q$ is thus a $\left(\lambda^{\prime}, 2 \lambda-2\right)$ correspondence, and the number of united tangents is therefore $\lambda^{\prime}+2 \lambda-2$, or the curves $R, \Delta$ touch in $\lambda^{\prime}+2 \lambda-2$ points.

The tangents $T, P$ each correspond to $T^{\prime}$, and they therefore correspond to each other. For a given position of $T$ we have a single position of $T^{\prime}$, and therefore $2 \lambda-2$ positions of $A$, and thence $(2 \lambda-2)(\lambda-2)$ positions of $P$; that is, for a given position of $T$ we have $(2 \lambda-2)(\lambda-2)$ positions of $P$. Again, to a given position of $P$ correspond $2 \lambda-4$ positions of $A$, therefore $(2 \lambda-4) \lambda^{\prime}$ positions of $T^{\prime \prime}$ or of $T$; that is, for a given position of $P$ we have $(2 \lambda-4) \lambda^{\prime}$ positions of $T$. The correspondence between $T, P$ is thus a $\left[2 \lambda^{\prime}(\lambda-2), 2(\lambda-1)(\lambda-2)\right]$ correspondence, and the number of united tangents is $2\left(\lambda+\lambda^{\prime}-1\right)(\lambda-2)$; or the curves $R, \Delta$ meet in $2\left(\lambda+\lambda^{\prime}-1\right)(\lambda-2)$ points.

Reckoning the contacts twice, the total number of intersections of $R, \Delta$ is

$$
2 \lambda^{\prime}+4 \lambda-4+2\left(\lambda+\lambda^{\prime}-1\right)(\lambda-2),=\left(\lambda+\lambda^{\prime}\right)(2 \lambda-2),
$$

as it should be.

In the particular case $\lambda=\lambda^{\prime}=2$, the curves $\Delta, \Delta^{\prime}$ are conics, and the curve $R$ is a quartic curve touching each of the conics 4 times; this is at once verified, since the equations here are

$$
a c-b^{2}=0, \quad a^{\prime} c^{\prime}-b^{\prime 2}=0, \quad 4\left(a c-b^{2}\right)\left(a^{\prime} c^{\prime}-b^{\prime 2}\right)-\left(a c^{\prime}+a^{\prime} c-2 b b^{\prime}\right)^{2}=0 .
$$

C. IX. 